\title{
104. A Note on the Theory of Electrical Phenomena in Imperfect Dielectric Substances.
}

\author{
By Heiichi Nukryama.
}

Institute of Electrical Engineering, Tohoku Imperial University, Sendai.

(Rec. June 25, 1926. Comm. by H. Nagaoka, M.r.a., July 12, 1926.)

1. Local Transient. Let $\%$ be the specific conductivity, $K$ the inductive capacity and $\theta$ the clectric intensity (the dot below the letter indicates a space vector), then in a space where

$$
A=\frac{\eta}{K}=\text { constant }
$$

is everywhere constant, the local transient may be expressed by the well known equation

$$
\operatorname{div} K G=\{\operatorname{div} K G\}_{t \rightarrow \infty} \varepsilon^{-4 \pi A t}
$$

and

$$
\tau=\frac{1}{4 \pi A}=\frac{K}{4 \pi \eta}
$$

is the relaxation time of the medium.

2. Stationary State. When the stationary state is reached, we have by. Eq. (2)

$$
\operatorname{div} K G=0
$$

and from Eq. (1) (4), we have also

$$
\operatorname{div} \geqslant G=0
$$

From these two equations we can show that the solution of the electric field is unique for a suitable boundary condition as a conduction electric current field.

3. Perfect Conductors in Conducting Dielectric Medium. If $l$ perfect conductors are placed in conducting dielectric medium satisfying Eq. (1), the law of superposition holds. The charge $Q$ of the conductor and the conduction electric current $I_{0}$ from the conductor to the dielectric 
medium may be expressed as linear functions of the potential $V$ of the conductors.

$$
\begin{array}{llll}
Q_{k} & =q_{k 1} V_{1}+q_{k 2} V_{2}+\cdots \cdots+q_{k k} V_{k}+\cdots \cdots+q_{k l} V_{l} & & (k=1,2, \cdots \cdots l) \\
I_{c k} & =g_{k 1} V_{1}+g_{k 2} V_{2}+\cdots \cdots+g_{l k} V_{k}+\cdots \cdots+g_{k l} V_{l} & (k=1,2, \cdots \cdots l)
\end{array}
$$

and we have here

$$
q_{1 k}=q_{k^{\prime},}, g_{i k}=g_{k^{\prime},}, g=4-A q
$$

4. Free Discharge. When the conductors are left free to discharge, the law of discharge is the same for all conductors and may be expressed by the following equation.

$$
Q_{k}=\left(Q_{k}\right)_{t=9} \varepsilon^{-4 \pi A t} \quad(k=1,2, \cdots \cdots l)
$$

When the potential of the conductors are given, there is no transient phenomena.

5. Periodic Steady State. When the potential of the conductors is harmonic

$$
\dot{V}_{k}=\sqrt{2} \dot{V}_{k}{ }^{j \omega t} \quad(k=1,2, \cdots \cdots l)
$$

(the dot above the letter indicates a complex number) the complex effective value of the current flowing from the conductor to the conducting dielectric medium is

$$
\begin{gathered}
\dot{I}_{k}=(4 \pi A+j \omega)\left(q_{k 1} \dot{V}_{1}+q_{k 2} \dot{V}_{2}+\cdots \cdots+q_{k i} \dot{V}_{k}+\cdots \cdots+q_{k l} \dot{V}_{l}\right. \\
(k=1,2, \cdots \cdots l)
\end{gathered}
$$

the average electrostatic energy $\bar{U}$ stored in the dielectrics is

$$
\bar{U}=\frac{1}{2} \sum_{k=1}^{l} \dot{q}_{k k} V_{k}^{2}+\frac{1}{2} \sum_{(k h)}^{\frac{1}{2} l(l-1)} q_{k h}\left(\dot{V}_{h} \bar{V}_{k}+\bar{V}_{h} \dot{V}_{k}\right)
$$

The vector power $\dot{P}$ given to the dielectric medium is

$$
\dot{P}=\left(\delta_{\pi} A-j 2 \omega\right) \bar{U}=2 \omega\left(\frac{1}{\omega \tau}-j\right) \bar{U}
$$

6. Dielectric Hystercsi. If we take

$$
(\dot{K})=\left(\dot{K}_{x x}, \dot{K}_{y y}, \dot{K}_{z z}, \dot{K}_{x y}, \dot{K}_{z y}, \dot{K}_{x: c}\right)
$$

as the complex tensor whose tensor vector product with the cornplex effective value of electric intensity $\dot{G}$ is the complex effective value of electric displacement, the vector power $\dot{P}_{d}$ given to the electric displacement field is 


$$
\begin{aligned}
\dot{P}_{a} & =\left(\left[\dot{G}, \frac{j \omega}{4 \pi} \dot{D}\right]_{p}\right)=-\frac{j \omega}{4 \pi}\left([\dot{G},(\dot{K}) \dot{G}]_{p}\right) \\
& =-\frac{j \omega}{4 \pi}\left(\left[\operatorname{tens}[\dot{G} \dot{G}]_{n},(\dot{K})\right]_{p}\right)
\end{aligned}
$$

The complex average electrostatic energv density $\dot{\bar{U}}$ is

$$
\dot{\bar{U}}=\frac{1}{S_{\pi}}\left([\dot{G}, \dot{D}]_{p}\right)=\frac{1}{8 \pi}\left(\left[\operatorname{tens}[\dot{G} \dot{G}]_{p},(\dot{K})\right]_{p}\right)
$$

where [ $]_{3}$, represents vector power product. From Eq. (13) (14)

$$
\dot{P}_{d}=-j 2 \omega \dot{\bar{U}}
$$

7. Apparent Cornplex Inductive Capacity. In a simple case when

$$
\left.\begin{array}{l}
\dot{K}_{x y}=\dot{K}_{y z}=\dot{K}_{2:}=0 \\
\dot{K}=\dot{K}_{x x}=\dot{K}_{y y}=\dot{K}_{z:}
\end{array}\right\}
$$

the apparent complex inductive capacity $\dot{K^{\prime}} \dot{\text { is }}$

$$
\dot{K}^{\prime}=\left(1-j \frac{4 \pi \overline{\dot{A}}}{(1)}\right) \dot{K}
$$

where

$$
\dot{A}=\frac{\eta}{\dot{\bar{K}}}=\text { constant }
$$

8. Stationary State in General Conducting Dielectric Medium. When the condition given by $\mathrm{Eq}$. (1) no longer holds, the electric field is determined by the condition

$$
\operatorname{div} \geqslant \nabla V=0
$$

and the space density $\rho$ of the true electric charge is

$$
\rho=-\frac{\gamma}{4 \div}\left(\nabla \Gamma, \nabla \frac{1}{A}\right)
$$

9. Periodic Steady State in General Conducting Dielectric Medium. The fundamental equation which determines the electric field in periodic steady state may be shown to be

$$
\operatorname{div} \eta \nabla \dot{y}=\sum_{j-\omega \tau}^{y}(\nabla \dot{j} \cdot \nabla \omega \tau)
$$

which generates to the ordinary Laplace's equation whon or is very sinall or very large. 
10. Permanent Polarization and the Fixed True Electric Charge. The permanent polarization may be distinguished from the fixed truf electric charge of Eq. (20) by the determination of the tensor ellipsoid

$$
r=-\frac{n}{\sqrt{\sigma^{\prime \prime}}}
$$

where $r$ is the radius vector of the tensor ellipsoid, $n$ the unit vector in an arbitrary direction, $\sigma^{\prime \prime}{ }_{\text {on }}$ the resultant surface charge of a thin disc whose plane is perpendicular to $n$.

The complete paper will appear in the Journal of the Institute of Electrical Engineers of Japan. 